\title{
A Novel Cell Morphology Analyzer Application in Head and Neck Cancer
}

\author{
Yongli Zhang $\mathbb{D}^{1,2}$ \\ Mengyao $\mathrm{Xie}^{1,2}$ \\ Ruoyan Xue ${ }^{1,2}$ \\ Qi Tang ${ }^{1,2}$ \\ Xiaohui Zhu',2 \\ Jian Wang $\mathbb{D}^{1,2}$ \\ Hua Yang $\mathbb{D}^{1,2}$ \\ Chao $\mathrm{Ma} \mathbb{( D}^{3-5}$
}

'Department of Otolaryngology, Chinese Academy of Medical Sciences and Peking Union Medical College, Peking Union Medical College Hospital, Beijing, 100730, People's Republic of China; ${ }^{2}$ Translational Medicine Center, Peking Union Medical College Hospital, Beijing, I00730, People's Republic of China; ${ }^{3}$ Institute of Basic Medical Sciences, Department of Human Anatomy, Histology and Embryology, Neuroscience Center, Chinese Academy of Medical Sciences, School of Basic Medicine, Peking Union Medical College, Beijing, I00005, People's Republic of China; ${ }^{4}$ Joint Laboratory of Anesthesia and Pain, Peking Union Medical College, Beijing, 100730, People's Republic of China; ${ }^{5}$ Chinese Institute for Brain Research, Beijing, 102206, People's Republic of China
Correspondence: Jian Wang; Hua Yang Department of Otolaryngology, Peking Union Medical College Hospital, No. I Shuaifuyuan Wangfujing Dongcheng District, Beijing, 100730, People's Republic of China Tel +86 |3673 |6426I; +86 I370 I I 27757

Fax+86-|0-69|563|I

Email wangjianent@163.com;

dr_yanghua202I@I63.com
Purpose: Rapid and accurate diagnosis of the pathological characteristics of head and neck cancer and tumor resection margins is important. The DiveScope cell morphology analyzer (DiveScope) is a new endomicroscope that can rapidly image living tissues and cells. In this study, we preliminarily examined the accuracy of the DiveScope for determining the malignancy of head and neck cancers and the positivity/negativity of tumor resection margins and determined the consistency between diagnostic results with the DiveScope and those of frozen section pathology to provide a foundation for further clinical trials in pathological diagnosis of head and neck cancers and tumor resection margins.

Methods: Head and neck cancer samples and resection margin samples were rapidly stained ex vivo before observation under the DiveScope cell morphology analyzer. Experienced pathologists distinguished the benignity and malignancy of the tumors based on images obtained by the DiveScope in a double-blind manner to validate the diagnostic performance of the analyzer.

Results: We found that the cell morphology, cell nucleus morphology, karyoplasmic ratio, and even the nucleolus could be clearly distinguished. The positive likelihood ratio (PLR) and negative likelihood ratio (NLR) of benign and malignant head and neck cancer according to DiveScope results were 10.55 and 0.04 , respectively. The PLR and NLR of the head and neck cancer resection margins according to the DiveScope were 19.01 and 0 , respectively.

Conclusion: The DiveScope showed high accuracy in determining the benignity and malignancy of head and neck cancer and the positivity/negativity of resection margins, and its results were highly consistent with those of intraoperative frozen section pathology tests. Keywords: the DiveScope cell morphology analyzer, head and neck cancer, resection margins, pathological diagnosis

\section{Introduction}

Head and neck cancer is one of the most common malignancies, and approximately $90 \%$ of cases are squamous cell carcinoma of the head and neck (HNSCC). ${ }^{1-3}$ Surgery is the primary method for treating head and neck cancer. Determination of the benignity and malignancy of head and neck cancer is vital for formulating a treatment plan, while determining the nature of the resection margin during surgery is vital for ensuring radical resection of $\mathrm{HNSCC}$, improving patient prognosis, and increasing the organ preservation rate. ${ }^{4-6}$ At present, histopathological examination is the most reliable method for determining the benignity and malignancy of head and neck cancer and the positivity/negativity of resection margins (including intraoperative frozen pathological examination and postoperative routine pathological examination). In addition, narrow band imaging (NBI), contact endoscopy, and optical coherence tomography can 
also aid in determining the nature of the lesion. ${ }^{7-9}$ Histopathological diagnosis is time-consuming and is unable to directly determine the surgical area during surgery. Although other auxiliary methods are less time-consuming, they have lower accuracy and cannot replace pathological examination. Therefore, these methods also have a weak ability to define the surgical area. In view of these shortcomings, a rapid and accurate technique for diagnosing lesions and their resection margins is needed. This technique will be able to help surgeons determine the lesion area during surgery without delaying surgery to wait for pathology results. The application of this technique will markedly change the process of HNSCC surgery, which is important for the diagnosis and treatment of head and neck cancer. The DiveScope cell morphology analyzer is a new endomicroscope that has the potential to bring us closer to this goal. The device uses real-time imaging and ultrahigh magnification combined with ultrahigh spatial resolution to achieve advanced microscopy imaging results and enable intraoperative live-cell imaging. However, no studies on the application potential and limitations of this microscope for head and neck cancer have been published. Hence, we conducted this study to explore the consistency of images acquired using this endomicroscope, images from routine pathology, and images from frozen pathology, thus providing a foundation for future studies.

\section{Materials and Methods}

The study included 47 patients with throat diseases who were treated with surgery performed by the same surgeon from December 2019 to September 2020. The study subjects were continuously enrolled, and detailed information was collected from all patients, including name, sex, age, clinical diagnosis, and lesion site.

The DiveScope cell morphology analyzer is an ultrahigh resolution miniaturized epifluorescence microscope that was independently developed by Micro Control Instruments, Ltd (Nanjing, China). Its lens length is $18 \mathrm{~cm}$ (maximum: $35 \mathrm{~cm}$ ), its lens diameter is $3 \mathrm{~mm}$, its resolution is $0.5 \mu \mathrm{m}$, the total magnification is $500 \mathrm{X}$, and it allows cells to be observed up to a depth of $50 \mu \mathrm{m}$ (Figure 1). The DiveScope cell morphology analyzer used in this study is the first-generation DiveScope (DSDemo). The DiveScope cell morphology analyzer is currently in the preclinical stage; thus, this article reports an in vitro study of the DiveScope cell morphology analyzer in head and neck tumors.

The image acquisition method was as follows: (1) Fresh $3 * 3-\mathrm{mm}$ head and neck cancer samples were collected. (2) After the samples were collected, they were immediately sprayed with $0.25 \%$ fluorescein sodium salt (Sigma-Aldrich, Inc., USA) solution for 2 minutes and washed with physiological saline. (3) Next, the samples were sprayed with $4 \%$ methylene blue (Sigma-Aldrich Inc, USA) solution for staining for 2 minutes, followed by washing with physiological saline. (4) An otolaryngologist used the DiveScope cell morphology analyzer to directly acquire images of the stained samples. (5) Two pathologists with extensive experience in head and neck cancer diagnosis performed diagnoses based on the acquired images. Differing opinions were resolved by discussion until a consensus regarding the diagnosis was reached. (6) Pathologists from Peking Union

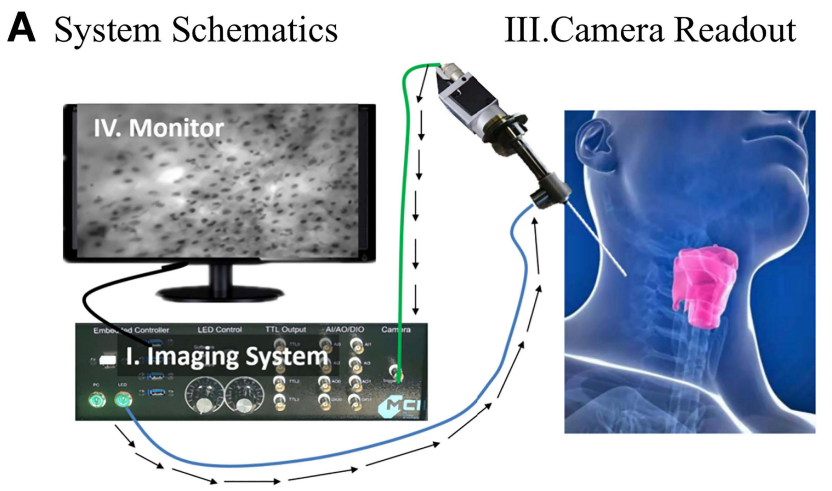

II.Excitation Light Source(LED)
B Optical Components

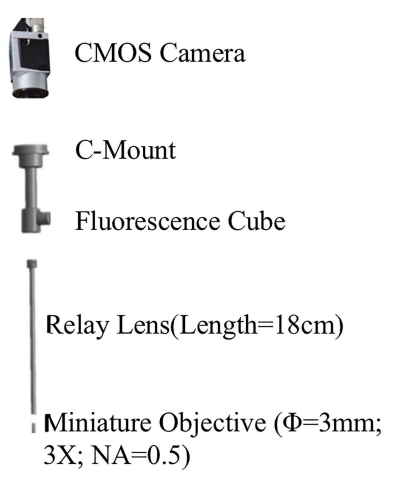

$3 \mathrm{X} ; \mathrm{NA}=0.5)$

Figure I Operating principle and composition of the DiveScope cell morphology analyzer system. (A) System schematics (components of the DiveScope cell morphology analyzer): (I) an image light source and an acquisition system, (II) an LED laser, (III) a camera for signal acquisition, and (IV) a display screen. The device can be used to perform tests at sites requiring observation during surgery, and the cell morphology and cell distribution in tissues can be observed on the display screen of the image acquisition system. (B) Core optics components: a miniaturized high-numerical-aperture objective lens, a relay lens, a fluorescence module, a $C$ port, and a camera. 
Medical College Hospital interpreted the frozen section pathology results and routine pathology results of tissues from the same site and determined a diagnosis. (7) The diagnostic results from steps (5) and (6) were compared and analyzed (Figure 2).

SPSS 20.0 was used to calculate the sensitivity, specificity, accuracy, positive predictive value (PPV), negative predictive value (NPV), positive likelihood ratio (PLR), and negative likelihood ratio (NLR) and their $95 \%$ confidence intervals (95\% CIs). Kappa values were calculated for consistency analysis.

The data analysis in this study was divided into three main parts. In part 1, the accuracy of the DiveScope cell morphology analyzer for determining the benignity and malignancy of head and neck cancer (compared with routine pathology results) was analyzed. In part 2, the accuracy of the DiveScope cell morphology analyzer for determining the positivity/negativity of head and neck cancer resection margin samples (compared with routine pathology results) was analyzed. In part 3 , the consistency between the DiveScope cell morphology analyzer and intraoperative frozen section pathological examinations in determining the benignity and malignancy of head and neck cancer and the positivity/negativity of head and neck cancer resection margin samples was assessed. In part 1 , postoperative routine pathology results of patients were used as the gold standard to determine the numbers of true-positive (TP), true-negative (TN), false-positive (FP), and false-negative (FN) results among the DiveScope cell morphology analyzer imaging results. These values were used to calculate the sensitivity, specificity, accuracy, PPV, NPV, PLR, and NLR of the DiveScope cell morphology analyzer in determining the benignity and malignancy of head and neck cancer. In part 2 , the patients' postoperative resection margin pathology results were used as the gold standard, and an analytical method similar to that used in part 1 was used to analyze the accuracy of the DiveScope cell morphology analyzer for determining the positivity/negativity of the resection margins. In part 3, kappa values were calculated to analyze the consistency of the DiveScope cell morphology analyzer and intraoperative frozen section pathology in determining the benignity and malignancy of head and neck cancer and the positivity/negativity of resection margins.

\section{Results}

A total of 47 patients were enrolled in this study. Their lesions were located in the oropharynx, lower pharynx, and larynx. The patients included 39 males and 8 females with a mean age was $55 \pm 12$ years, and 22 and 25 cases were benign and malignant, respectively (Supplementary Table 1). Parallel DiveScope cell morphology analyzer examinations, frozen section pathological examinations, and routine pathological examinations were carried out on lesion tissues from 47 patients and resection margin tissues from 20 patients.

The images obtained from the DiveScope cell morphology analyzer were pleasing, clearly showing the morphology and distribution characteristics of stained cell nuclei and cytoplasm in tissue samples (including healthy tissue, lesion tissue, and resection margin tissue). Therefore, this device is suitable for use in pathological

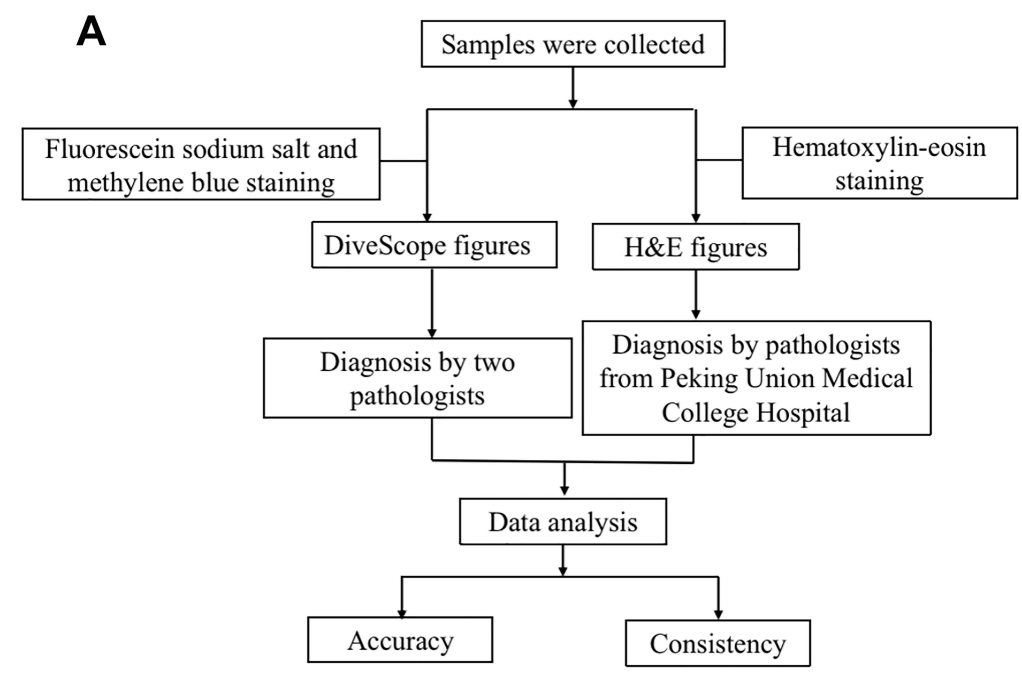

B

Figure 2 Work flow chart. (A) Experiment flow chart. (B) Tissue preparation for DiveScope flow chart.

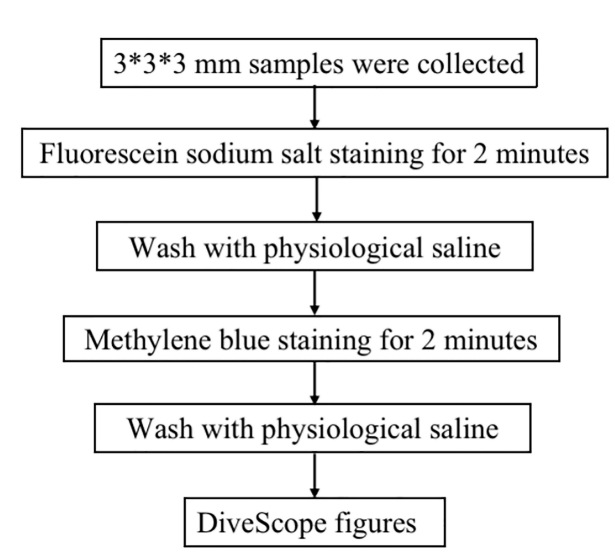


analyses. The characteristics of these images were very similar to those of routine pathology images (Figures 3-6).

Through data analysis and comparison of DiveScope cell morphology analyzer image interpretation results with routine hematoxylin-eosin (HE) pathology results, we found that the sensitivity, specificity, accuracy, PPV, and NPV of the degree of lesion tissue malignancy obtained from the DiveScope cell morphology analyzer were all above 90\%, the PLR was 10.55 (95\% CI: 5.37-20.73), and the NLR was 0.04 (95\% CI: 0.03-0.30) (Table 1). The sensitivity, specificity, accuracy, PPV, and NPV of the resection margin diagnosis determined by the DiveScope cell morphology analyzer were all above 90\%; the PLR was 19.01 (95\% CI: 7.18-50.32); and the NLR was 0 (95\% CI: -) (Table 2). A consistency analysis of the DiveScope cell morphology analyzer results and intraoperative frozen section pathology results showed that the kappa values for lesion tissue analyses and the lesion resection margin were 0.87 and 0.64 , respectively.

\section{Discussion}

The present study is the first to report the results of applying the DiveScope cell morphology analyzer to determine the benignity and malignancy of head and neck cancer and the resection margin characteristics. In this study, the high magnification and high resolution of the DiveScope cell morphology analyzer contributed to the extremely high clarity of the histopathology images. This clear, real-time cell imaging technology has no precedent in past papers. The sensitivity, specificity, and accuracy of the DiveScope cell morphology analyzer in determining the nature of head and neck cancers and resection margins were all greater than $90 \%$, indicating that the DiveScope cell morphology analyzer is highly accurate for diagnosing head and neck cancer. The PPV and NPV of the DiveScope cell morphology analyzer in determining the benignity and malignancy of head and neck cancer were greater than $90 \%$, the PLR was greater than 10 , and the NLR was greater than 0.1 , showing that the DiveScope cell

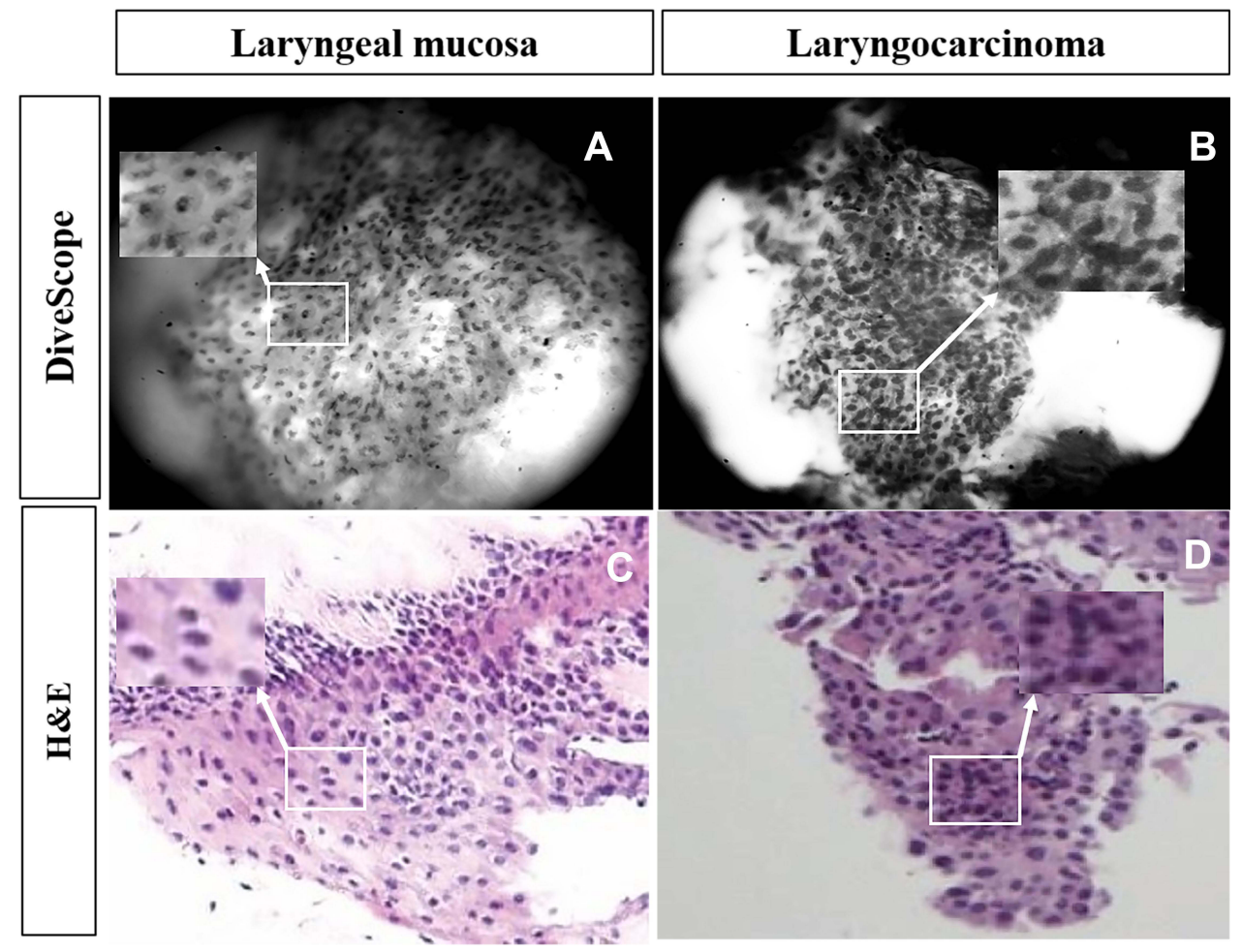

Figure 3 DiveScope images and hematoxylin-eosin (HE) staining pathology images of cells from healthy tissues and malignant tissues in the head and neck. Figures ( $\mathbf{A}$ and $\mathbf{C})$ are a DiveScope image and an HE staining pathology image of healthy mucosal epithelial tissues in the larynx, respectively. Figures (B and D) are a DiveScope image and an HE staining pathology image of laryngeal squamous cell carcinoma tissues. Figure (A) (Magnification: 500X): Healthy mucosal epithelium in the larynx. Cells and the cell nucleus morphology were regular and neatly arranged, the cell nucleus and cytoplasm were evenly stained, and the nucleolar structure could be observed in some cells (the white box shown in Figure (A). These characteristics are similar to those shown in Figure (C) (the white box shown in Figure (C). Figure (B) (Magnification: 500X): Squamous cell carcinoma tissues in the larynx. The cell nuclei were enlarged, deeply stained, irregular in morphology, and not neatly arranged (some nuclei were arranged together), showing typical pathological characteristics of squamous cell carcinoma (white box in figure (B). These characteristics are similar to those shown in Figure (D) (the white box shown in Figure (D). 


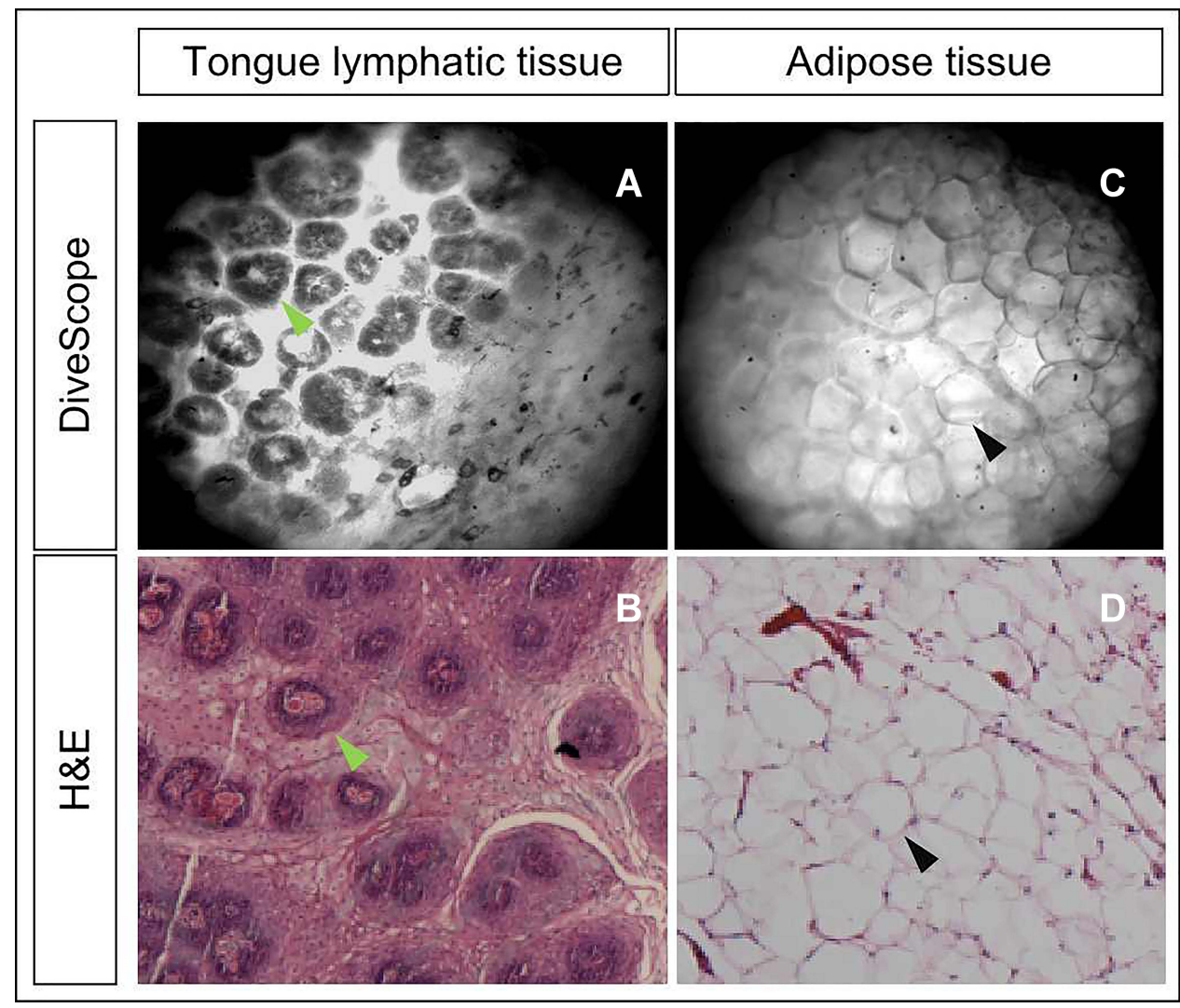

Figure 4 DiveScope images and hematoxylin-eosin (HE) staining pathology images of healthy tissue in the head and neck. Figure (A) and figure (B) are a DiveScope image and an HE-stained pathology image of tongue lymphatic tissues. Figure (C) and figure (D) are a DiveScope image and an HE-stained pathology image of adipose tissues. (A and B) show the lymphoid follicles (green arrows show) in the tongue lymphatic tissues. Figure (C) and figure (D) show transparent vacuoles in adipocytes and cell nuclei located at one side of the cytoplasm (black arrows show).

morphology analyzer can distinguish between benign tumors and malignant tumors of the head and neck. In this study, the PLR was $>10$ and the NLR was $<0.1$ when the DiveScope cell morphology analyzer was used to determine the nature of resection margins, suggesting that the DiveScope cell morphology analyzer can distinguish between positive and negative resection margins with high accuracy. However, the PPV of the DiveScope cell morphology analyzer in determining the nature of resection margins was 50\%. The reason for the low PPV is the low number of total samples used to determine the positivity/negativity of the resection margins. Therefore, more resection margin samples are required in the future for further studies. Comparative analysis with intraoperative frozen section pathology results showed that the kappa values of the DiveScope cell morphology analyzer in determining the degree of lesion malignancy and the positivity/negativity of resection margin tissues were 0.87 and 0.64 , respectively, suggesting that the DiveScope cell morphology analyzer has good consistency with intraoperative frozen section pathological examination.
The intraoperative diagnostic techniques currently used to determine the nature of head and neck cancer lesions include intraoperative frozen pathological examination, NBI, and contact endoscopy. For intraoperative frozen section pathological examinations, mass tissues were extracted and stained with $\mathrm{HE}$ for rapid determination of the nature of the mass. Currently, HE staining is the most widely used technique in clinical practice. However, this method requires 30 minutes to yield results and is characterized by sampling randomness. ${ }^{10-13}$ NBI can be used to determine the nature of a lesion through the microvascular morphology of the mucosal surface and has good application value in the early diagnosis of head and neck cancer. However, this technique uses the observed microvascular morphology to facilitate diagnosis; it cannot directly depict the cell structure of the mucosal surface ${ }^{7,14-17}$ or determine the degree of tumor malignancy at the cellular level. Contact endoscopy can be used to observe the cellular structure of head and neck mucosa and assist in minimally invasive pathological examination of laryngeal cancer and early-stage lesions. However, this technique has shortcomings such as low magnification, low resolution, and poor image quality. ${ }^{18-20}$ In 


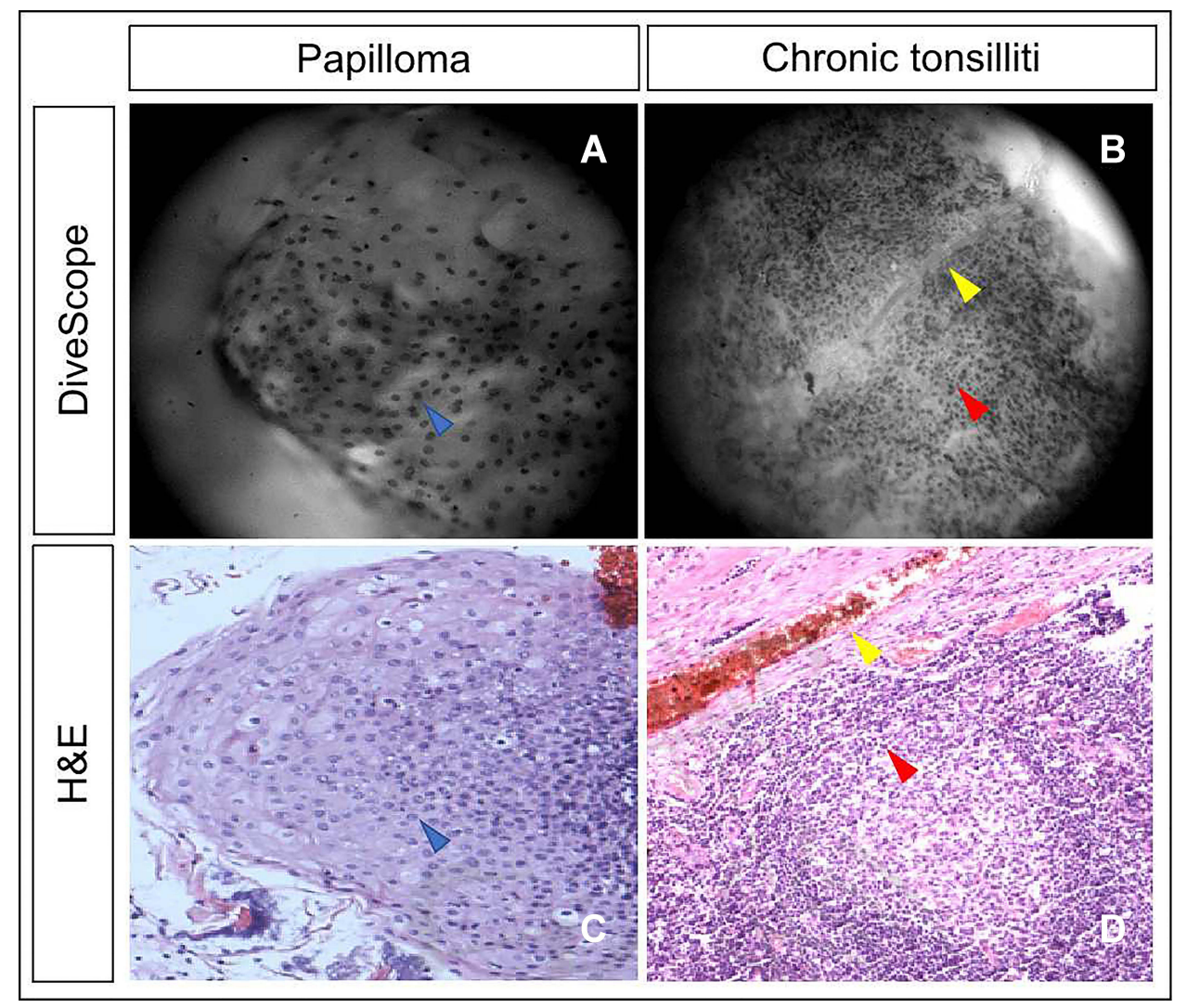

Figure 5 DiveScope images and hematoxylin-eosin (HE) staining pathology images of benign lesion tissues from the head and neck. Figures (A and $\mathbf{C})$ are a DiveScope image and an HE staining pathology image of laryngeal papilloma tissues. Figures (B and D) are a DiveScope image and an HE staining pathology image of tonsils in patients with chronic tonsillitis. Figures (A and $\mathbf{C}$ ) show squamous epithelial cells (blue arrows) in papilloma tissues and papillary growth of papilloma tissues. Figures (B) and (D) show many inflammatory cells (red arrows) and blood vessels.

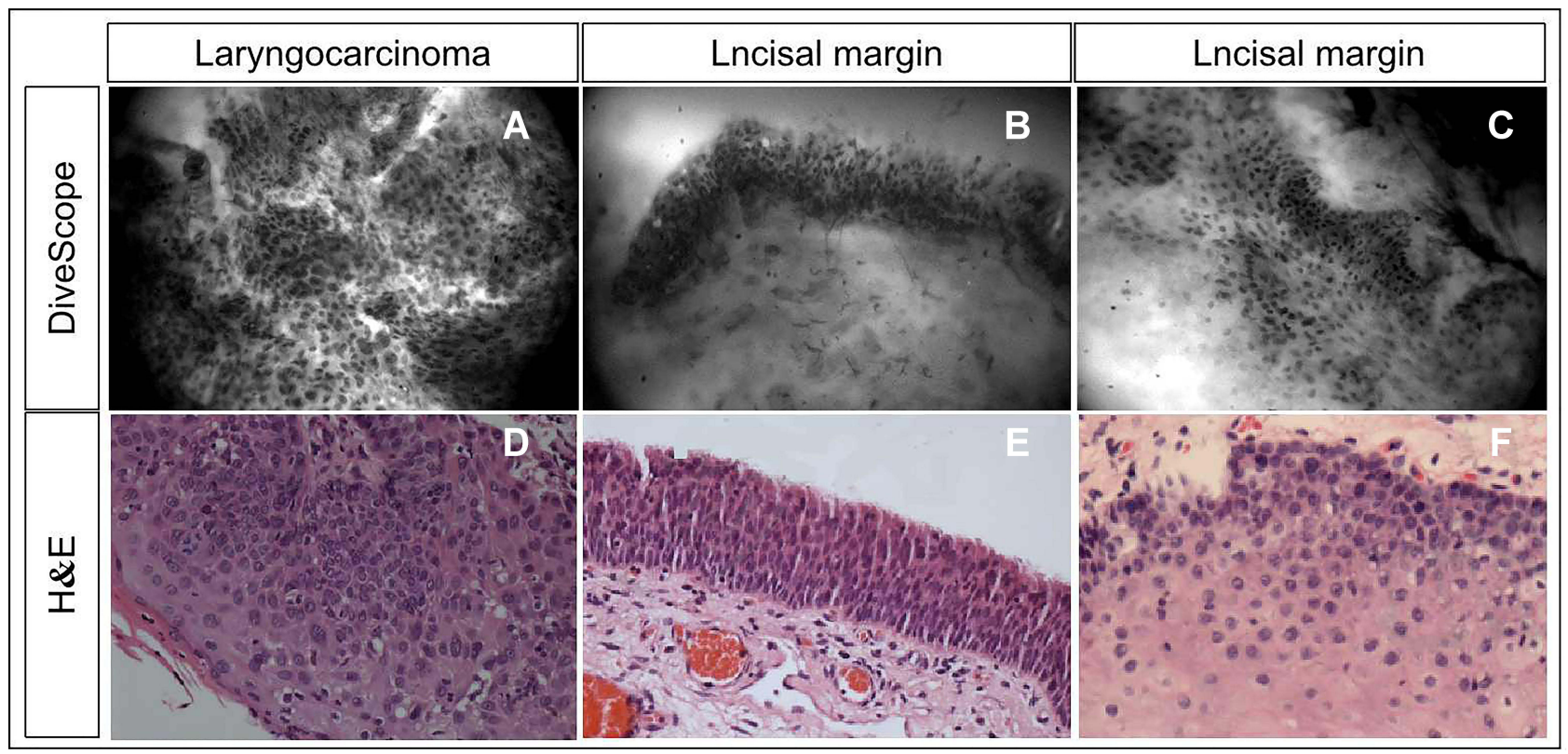

Figure 6 DiveScope images and hematoxylin-eosin staining pathology images of head and neck squamous cell carcinoma and resection margin tissues. Figures (A and D) show a DiveScope image and an HE staining pathology image of laryngeal cancer tissues. Figures (B, C, E, and F) show DiveScope images and HE staining pathology images of laryngeal cancer resection margin tissues. Figures ( $\mathbf{A}$ and $\mathbf{D})$ show the large nuclei, deep staining, and irregular morphology of laryngeal cancer cells. Figures (B and $\mathbf{E})$ show the regular morphology and neat arrangement of squamous epithelial cells in laryngeal cancer resection margin tissues and no heterotopic nuclei. Figures (C and F) show normal squamous epithelial cells and scattered inflammatory cells. 
Table I Analysis of the Accuracy of the DiveScope Cell Morphology Analyzer for Determining the Degree of Malignancy of Head and Neck Cancer $(n=47)$

\begin{tabular}{|l|c|}
\hline & $\mathbf{( 9 5 \% ~ C l )}$ \\
\hline Sensitivity & $96.00 \%(77.68-99.79 \%)$ \\
Specificity & $90.90 \%(69.38-98.41 \%)$ \\
Accuracy & $93.62 \%(81.44-98.34 \%)$ \\
PPV & $92.31 \%(73.4-98.66 \%)$ \\
NPV & $95.24 \%(74.13-99.75 \%)$ \\
PLR & $10.55(5.37-20.73)$ \\
NLR & $0.04(0.03-0.30)$ \\
\hline
\end{tabular}

Table 2 Analysis of the Accuracy of the DiveScope Cell Morphology Analyzer for Determining the Positivity/Negativity of Head and Neck Cancer Resection Margins ( $n=20)$

\begin{tabular}{|l|c|}
\hline & (95\% Cl) \\
\hline Sensitivity & $100 \%(5.46-100.00 \%)$ \\
Specificity & $94.74 \%(71.90-99.73 \%)$ \\
Accuracy & $95 \%(73.06-99.74 \%)$ \\
PPV & $50 \%(2.67-97.33 \%)$ \\
NPV & $100 \%(78.12-100.00 \%)$ \\
PLR & $19.01(7.18-50.32)$ \\
NLR & $0(-)$ \\
\hline
\end{tabular}

summary, existing intraoperative methods for determining the nature of a lesion have shortcomings such as long wait times, low clarity, and low accuracy. Therefore, a technique that can provide a fast and accurate diagnosis of a lesion and its resection margin will overcome these deficiencies. From the results of this study, we found that the DiveScope cell morphology analyzer can address the above technical shortcomings. This new cell microscope can be used to directly observe the cellular structure of tissues without sampling after quick staining of living tissues with spraying (which requires approximately 5 minutes to yield results); thus, it can rapidly and accurately determine the degree of tissue malignancy. In a comparative analysis with intraoperative frozen pathology results, we found that the results obtained with the DiveScope cell morphology analyzer and those obtained by intraoperative frozen section pathological examination have a high degree of consistency and that the former can be used in the future to directly guide the surgeon in determining the resection area during surgery, decreasing both the risk of an insufficient postoperative resection margin and excessive resection, which affects postoperative quality of life and can maximize organ function preservation.
This study had some limitations. This was an ex vivo clinical trial, and further in vivo studies may be necessary. In the future, we will collect more samples and conduct in vivo studies to provide additional evidence to support the feasibility of employing the DiveScope cell morphology analyzer for head and neck cancer diagnosis. We hope that the device can be used in clinical applications in the future for rapid and accurate pathological diagnosis of head and neck cancer.

\section{Conclusion}

The DiveScope cell morphology analyzer has high accuracy in the pathological diagnosis of head and neck tumors and may become a new type of technology with considerable clinical application potential that can facilitate rapid intraoperative pathological diagnosis.

\section{Ethics Approval and Informed Consent}

This study was approved by the ethics committee of Peking Union Medical College Hospital (ethics approval no. JS2084), conducted in accordance with the Declaration of Helsinki, and all patients signed informed consent forms.

\section{Acknowledgments}

We would like to thank the Micro Control Instruments Ltd. for their device (the DiveScope cell morphology analyzer) assistance.

\section{Funding}

This work was supported by clinical research on a new kind of tympanic ventilation tube (2019XK320021), the National Key Research and Development Plan of China (2020YFC2005200), the National Key Research and Development Plan of China (2016YFA0101000, 2016YFA0101002), and the Nature Science Foundation of Beijing, China (7192171).

\section{Disclosure}

The authors declare that they have no conflicts of interest.

\section{References}

1. Chow LQM. Head and neck cancer. N Engl J Med. 2020;382 (1):60-72. doi:10.1056/NEJMra1715715

2. Rettig EM, D'Souza G. Epidemiology of head and neck cancer. Surg Oncol Clin N Am. 2015;24(3):379-396. doi:10.1016/j.soc.2015.03.001

3. Vigneswaran N, Williams MD. Epidemiologic trends in head and neck cancer and aids in diagnosis. Oral Maxillofac Surg Clin North Am. 2014;26(2):123-141. doi:10.1016/j.coms.2014.01.001 
4. Thomas Robbins K, Triantafyllou A, Suárez C, et al. Surgical margins in head and neck cancer: intra- and postoperative considerations. Auris Nasus Larynx. 2019;46(1):10-17. doi:10.1016/j.anl.20 18.08.011

5. Magliocca KR. Surgical margins: the perspective of pathology. Oral Maxillofac Surg Clin North Am. 2017;29(3):367-375. doi:10.1016/j. coms.2017.05.002

6. Dyalram D, Caldroney S, Heath J, et al. Margin analysis: cutaneous malignancy of the head and neck. Oral Maxillofac Surg Clin North Am. 2017;29(3):341-353. doi:10.1016/j.coms.2017.04.001

7. Ni XG, Wang GQ. The role of narrow band Imaging in head and neck cancers. Curr Oncol Rep. 2016;18(2):10. doi:10.1007/s11912-0150498-1

8. Hughes OR, Stone N, Kraft M, Arens C, Birchall MA. Optical and molecular techniques to identify tumor margins within the larynx. Head Neck. 2010;32(11):1544-1553. doi:10.1002/hed.21321

9. Thompson LD. Diagnostically challenging lesions in head and neck pathology. Eur Arch Otorhinolaryngol. 1997;254(8):357-366. doi:10.1007/BF01642550

10. Dimery IW, Hong WK. Overview of combined modality therapies for head and neck cancer. J Natl Cancer Inst. 1993;85(2):95-111. doi:10.1093/jnci/85.2.95

11. Hoerbelt R, Padberg W. Primäre trachealtumoren in hals und mediastinum: resektions- und rekonstruktionsverfahren [Primary tracheal tumors of the neck and mediastinum: resection and reconstruction procedures]. Chirurg. 2011;82(2):125-133. German. doi:10.1007/ s00104-010-1974-7

12. Spector ME, Farlow JL, Haring CT, Brenner JC, Birkeland AC. The potential for liquid biopsies in head and neck cancer. Discov Med. 2018;25(139):251-257.
13. von Buchwald C, Bilde A, Shoaib T, Ross G. Sentinel node biopsy: the technique and the feasibility in head and neck cancer. $O R L$ J Otorhinolaryngol Relat Spec. 2002;64(4):268-274. doi:10.1159/ 000064143

14. Zhou H, Zhang J, Guo L, Nie J, Zhu C, Ma X. The value of narrow band imaging in diagnosis of head and neck cancer: a meta-analysis. Sci Rep. 2018;8(1):515. doi:10.1038/s41598-017-19069-0

15. Lin YC, Watanabe A, Chen WC, Lee KF, Lee IL, Wang WH. Narrowband imaging for early detection of malignant tumors and radiation effect after treatment of head and neck cancer. Arch Otolaryngol Head Neck Surg. 2010;136(3):234-239. doi:10.1001/archoto.2009.230

16. Lee CT, Chang CY, Lee YC, et al. Narrow-band imaging with magnifying endoscopy for the screening of esophageal cancer in patients with primary head and neck cancers. Endoscopy. 2010;42 (8):613-619. doi:10.1055/s-0030-1255514

17. Garofolo S, Peretti G, Piazza C. Role of narrow-band imaging in detection of head and neck unknown primary squamous cell carcinoma. Laryngoscope. 2018;128(9):2060-2066. doi:10.1002/lary.27098

18. Pavlidis $\mathrm{P}$, Gouveris $\mathrm{H}$, Gorgulla $\mathrm{H}$, Hast HJ, Maurer J. Electrogustometry and contact endoscopy findings in patients with head and neck malignancies treated with chemotherapy, radiotherapy, or radiochemotherapy. Chem Senses. 2015;40(3):165-171. doi:10.1093/chemse/bju060

19. Warnecke A, Averbeck T, Leinung M, et al. Contact endoscopy for the evaluation of the pharyngeal and laryngeal mucosa. Laryngoscope. 2010;120(2):253-258. doi:10.1002/lary.20732

20. Dedivitis RA, Pfuetzenreiter EG Jr, Guimarães AV. Contact endoscopy of the larynx as an auxiliary method to the surgical margins in frontolateral laryngectomy. Acta Otorhinolaryngol Ital. 2009;29 (1):16-20.
International Journal of General Medicine

\section{Publish your work in this journal}

The International Journal of General Medicine is an international, peer-reviewed open-access journal that focuses on general and internal medicine, pathogenesis, epidemiology, diagnosis, monitoring and treatment protocols. The journal is characterized by the rapid reporting of reviews, original research and clinical studies

\section{Dovepress}

across all disease areas. The manuscript management system is completely online and includes a very quick and fair peer-review system, which is all easy to use. Visit http://www.dovepress.com/ testimonials.php to read real quotes from published authors. 